\title{
The Sixth Annual Translational Stem Cell Research Conference of the New York Stem Cell Foundation
}

\author{
Caroline Marshall, ${ }^{1}$ Haiqing Hua, ${ }^{1,2}$ Linshan Shang, ${ }^{1}$ Bi-Sen Ding, ${ }^{3,1}$ Giovanni Zito, ${ }^{4,1}$ \\ Giuseppe Maria de Peppo, ${ }^{1}$ George Kai Wang, ${ }^{2,1}$ Panagiotis Douvaras, ${ }^{1}$ Andrew A. Sproul, ${ }^{1}$ \\ Daniel Paull, ${ }^{1}$ Valentina Fossati, ${ }^{1}$ Michael W. Nestor, ${ }^{1}$ David McKeon, ${ }^{1}$ Kristin A. Smith, ${ }^{1}$ \\ and Susan L. Solomon ${ }^{1}$ \\ ${ }^{1}$ The New York Stem Cell Foundation, New York, New York. ${ }^{2}$ Columbia University, New York, New York. ${ }^{3}$ Weill Cornell Medical \\ College, New York, New York. ${ }^{4}$ Yale University, New Haven, Connecticut
}

Address for correspondence: Caroline Marshall, The New York Stem Cell Foundation, 163 Amsterdam Avenue, Box 309, New York, NY 10023

The New York Stem Cell Foundation's “Sixth Annual Translational Stem Cell Research Conference” convened on October 11-12, 2011 at the Rockefeller University in New York City. Over 450 scientists, patient advocates, and stem cell research supporters from 14 countries registered for the conference. In addition to poster and platform presentations, the conference featured panels entitled "Road to the Clinic" and "The Future of Regenerative Medicine."

\section{Introduction}

The New York Stem Cell Foundation's (NYSCF) annual translational stem cell conference opened with two panels of experts discussing current progress in translating stem cell research to accelerate cures for the major diseases of our time. In the first panel "Road to the Clinic," moderated by Lee Rubin, director of translational medicine at the Harvard Stem Cell Institute and NYSCF scientific advisor, leading experts from the pharmaceutical, biotechnology, and healthcare industries were joined by representatives from venture capital and grant-awarding foundations to discuss how to transfer stem cell research from the laboratory into safe and effective treatments. Panelists included Stephen Chang (New York Stem Cell Foundation), Scott Johnson (Myelin Repair Foundation), Robert J. Palay (Cellular Dynamics International), and William A. Sahlman (Harvard Business School). The panel discussed the latest research developments and the scientifically challenging path toward clinical trials. The second panel, "The Future of Regenerative Medicine," composed of leading stem cell researchers and policy makers, discussed from their differing perspectives the regulatory challenges fac- ing researchers on the road to the clinic. Moderator and chief executive officer of the New York Stem Cell Foundation Susan L. Solomon, and panelists Mahendra S. Rao (NIH Intramural Center for Regenerative Medicine), Craig B. Thompson (Memorial Sloan-Kettering Cancer Center), Marc TessierLavigne (The Rockefeller University), and Irving L. Weissman (Stanford University) examined the changes in policy that will be needed to advance the development, evaluation, and approval of emerging stem cell treatments and regenerative medicine.

As in previous years, the second day of the conference convened an international panel of researchers at the forefront of the stem cell field who presented their work on diabetes, heart and muscles, cancer and blood disease, neurodegeneration, and programming/reprogramming. ${ }^{1,2}$ The panel included a groundbreaking report on somatic cell nuclear transfer (SCNT), recently published in Nature by Dieter Egli at the NYSCF laboratory.

In addition to a keynote address by Irving L. Weissman, who discussed the latest developments in cancer treatments using stem cells, the inaugural recipient of the NYSCF-Robertson Stem Cell Prize, Professor Peter J. Coffey (University College London, and the London Project to 
Cure Blindness) presented his forthcoming clinical trial using stem cells to treat age-related macular degeneration.

NYSCF, in collaboration with Annals of New York Academy of Sciences, is delighted to present this report; compiled by young stem cell scientists, it summarizes the excellent, groundbreaking progress in stem cell research.

\section{Diabetes}

The opening speaker, Hans Snoeck (Mount Sinai School of Medicine), discussed the importance of using stem cells to generate thymus tissue. In vivo, the thymus is the immune organ in which T cell positive and negative selection takes place. Thymus tissue derived in vitro has potential therapeutic applications, such as enhancing $T$ cell reconstitution after allogeneic bone marrow transplantation. Humanized mouse models can be created by transplanting thymus generated from human stem cells, which are extremely valuable for studying human autoimmune diseases, including type 1 diabetes. ${ }^{3}$ Snoeck showed that when using activin A, BMP4, and basic fibroblast growth factor (bFGF), his group differentiated human embryonic stem cells into anterior endoderm, from which thymic tissue is derived. Subsequent treatment with Noggin and SB-431542 directs endoderm cells toward anterior foregut fate, with more than $90 \%$ of the cells expressing SOX $2 .{ }^{4}$ By applying knowledge- and screening-based approaches, Snoeck's group discovered combinations of various factors, including Wnt3A, fibroblast growth factor 10 (FGF10), keratinocyte growth factor (KGF), and sonic hedgehog $(\mathrm{SHH})$, that could further specify foregut endoderm cells to become lung bud or pharyngeal endoderm cells. Notably, Snoeck pointed out that Wnt3A is crucial to prepattern the cells for lung development, and that retinoic acid induces lung fate partly through suppression of TBX1, a transcription factor necessary for pharyngeal development.

Shuibing Chen (Weill Cornell Medical College; Fig. 1) summarized previous and ongoing efforts to generate pancreatic $\beta$ cells from stem cells, including hypothesis-driven approaches based on animal models and in vitro studies that work well for the early stages of differentiation, specifically during the definitive endoderm and gut-tube endoderm stage. ${ }^{5}$ In order to efficiently direct the cells into pancreatic progenitors and $\beta$ cells, however, she and her colleagues have been using discoverydriven screen approaches to search for the optimal conditions to facilitate this transition. After screening with chemical libraries, they identified a small molecule, (-)-indolactam V, that can promote the generation of pancreatic progenitors by activating protein kinase $\mathrm{C}$ (PKC) signaling. ${ }^{6}$ Recently, Chen's group screened for growth factors and cell lines that induce pancreatic progenitor and $\beta$ cell generation and showed that mouse pancreatic endothelial cells and a human endothelial cell line (AKT-HBVEC) significantly enhance the proliferation of pancreatic progenitors in culture. Furthermore, Chen found that cell-cell contact is not required and the effect is mediated by factors that belong to the epidermal growth factor (EGF) family. Currently, the insulinproducing $\beta$ cells derived in vitro are immature $\beta$ cells, which do not display great response to glucose. Chen's group is now seeking a cellular niche that can accelerate the proliferation and/or maturation of $\beta$ cells.

The final talk in this session was given by Pedro Herrera (University of Geneva) who introduced the intriguing phenomenon of $\alpha$ cell to $\beta$ cell transition. Herrera's group has developed new transgenic models allowing near-total $\alpha$ cell or $\beta$ cell removal, specifically in adult mice; these mouse models enable the study of pancreas regeneration after massive cell loss. Herrera and colleagues showed that six months after $\beta$ cell removal, new $\beta$ cells were regenerated. Around $20 \%$ of newly generated $\beta$ cells express both glucagon and insulin, hormones produced by $\alpha$ cells and $\beta$ cells, respectively. Using celltracing technology, Herrera and colleagues demonstrated that some of the newly formed $\beta$ cells were derived from $\alpha$ cells. ${ }^{7}$ In contrast, they also observed that when most of $\alpha$ cells are removed, the remaining $2 \%$ of the normal $\alpha$ cell mass is enough to maintain healthy and euglycemic mice. ${ }^{8}$ Taken together, these studies suggest that future diabetic therapies could involve regenerating $\beta$ cells via reprogramming adult $\alpha$ cells.

\section{Cancer and blood disease}

Shahin Rafii (Howard Hughes Institute and Weill Cornell Medical College) opened this session and described a recently identified instructive role of pulmonary capillary endothelial cells (PCECs) in supporting lung regeneration. ${ }^{9}$ Previous work from the Rafii group established the novel concept that 


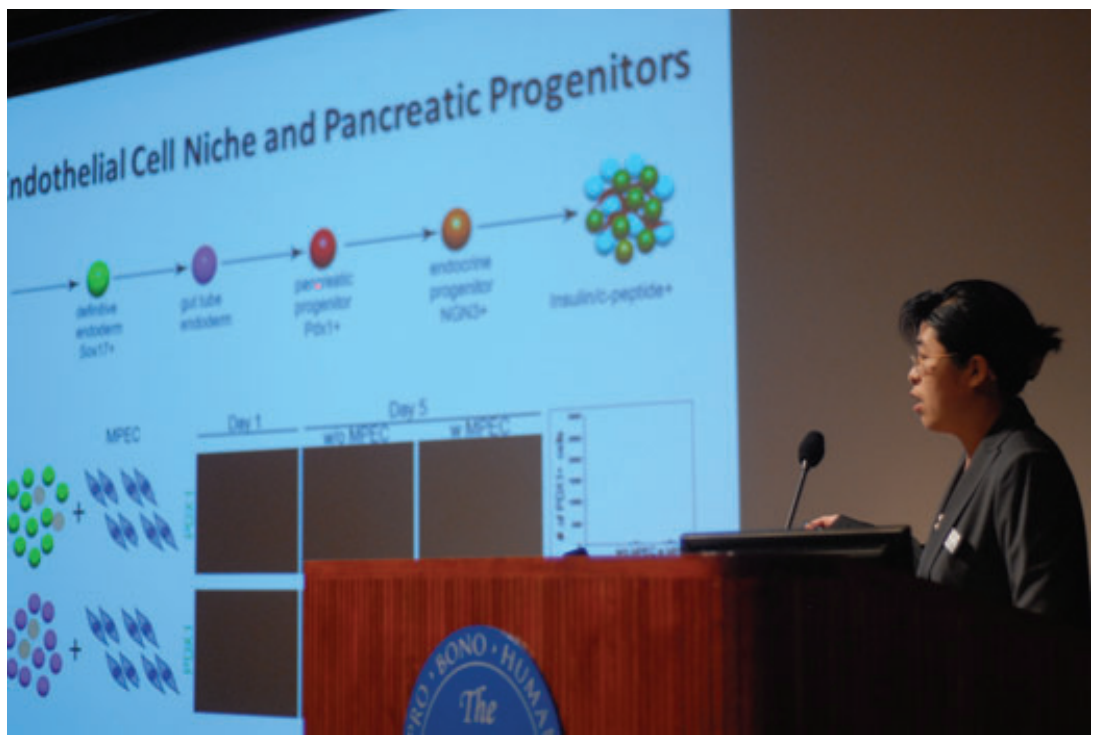

Figure 1. Shuibing Chen (Weill Cornell Medical College and NYSCF-Robertson investigator) presents her work on deriving functional pancreatic endocrine cells from human pluripotent stem cells.

capillary endothelial cells (CECs) not only function as passive conduits to meet metabolic demand, they also stimulate paracrine (angiocrine) growth factors to induce and sustain organ regeneration. ${ }^{10-14}$ In bone marrow $(\mathrm{BM})^{10-12}$ and liver, ${ }^{13}$ sinusoidal endothelial cells (SECs) constitute phenotypically and functionally distinct populations of cells. After partial hepatectomy, liver SECs-via angiocrine production of hepatocyte growth factor and Wnt2 (a process defined as inductive angiogenesis)—stimulate hepatocyte proliferation. ${ }^{13}$ Subsequently, liver SECs undergo proliferative angiogenesis to match the increasing demand for blood supply in the regenerating liver. Likewise, after chemotherapy and irradiation, activated BM SECs induce hematopoiesis by angiocrine generation of Notch ligands and insulin-like growth factor binding proteins (IGFBPs). ${ }^{10,12}$

To investigate the role of PCECs in supporting lung regeneration, Rafii's group generated a unilateral pneumonectomy (PNX) mouse model by performing surgical resection of the left lung lobe. Without perturbing the vascular integrity of the remaining lobes, PNX induces significant growth of mass, volume, and physiological respiratory capacity of the remaining lungs. This regeneration process is due to neoalveologenesis, a process involving amplification of alveolar epithelial progenitor cells and PCECs. The phenotypic and op- erational markers of mouse PCECs were defined as VE-cadherin ${ }^{+}$VEGFR2 $^{+}$FGFR $^{+}{ }^{+} \mathrm{CD}^{2} 4^{+}$ECs. They further demonstrated that PNX, through activation of VEGFR2 and FGFR1, induces PCECs of the remaining lobes to produce the angiocrine matrix metalloprotease MMP14. In turn, MMP14 promotes regenerative alveolarization by unmasking cryptic EGF-like ligands that stimulate proliferation of epithelial progenitor cells. These studies underscore the instrumental role of endothelial-derived angiocrine signals in instructing adult organ regeneration. Selective activation of CECs, or increasing the generation of angiocrine factors in patients, would promote organ regeneration, thereby offering therapeutic avenues for end-stage liver, lung, and hematopoietic diseases.

The second speaker of the session was Viviane Tabar (Memorial Sloan-Kettering Cancer Center), whose laboratory focuses on understanding the alterations within the brain stem cell niche that are responsible for glioblastoma tumors. Previously, it was demonstrated that a subpopulation of $\mathrm{CD} 133^{+}$ cancer stem cells are responsible for the initiation of glioblastoma tumors. ${ }^{15}$ Tabar's group discovered that $\mathrm{CD}_{133^{+}}$cancer stem cells not only initiate the tumor but also generate new endothelial cells necessary for tumor self-maintenance. ${ }^{16}$ The newly generated cells presented a different genotype when compared with normal blood vessel 


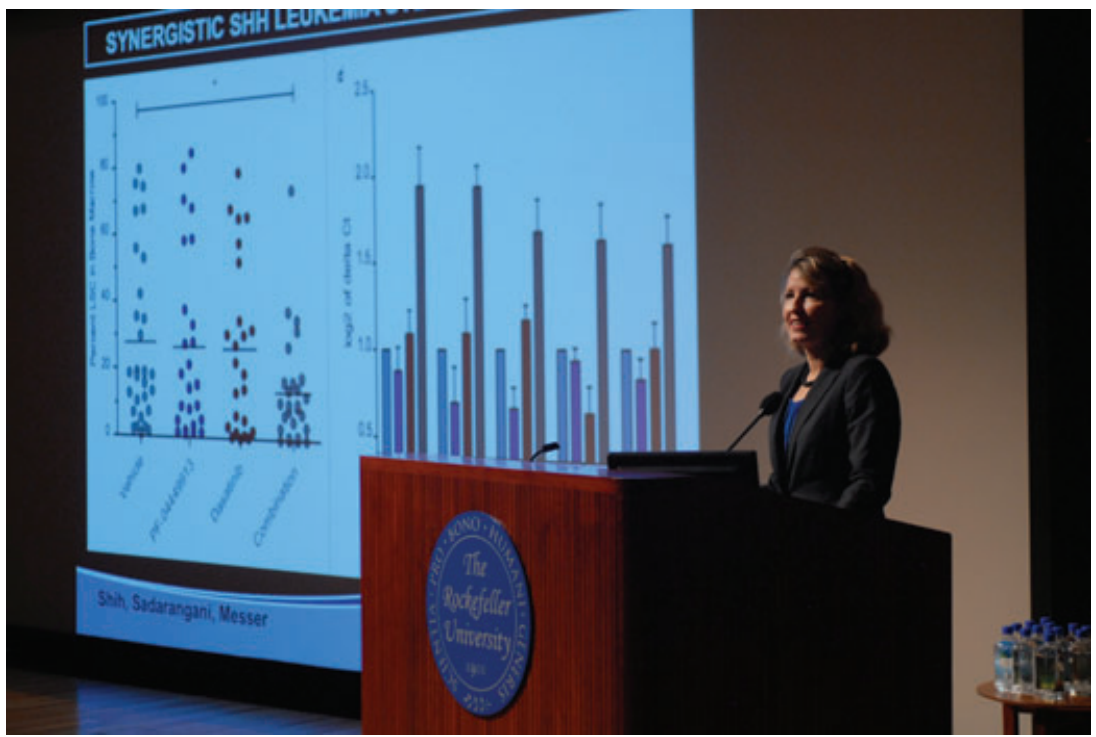

Figure 2. Catriona Jamieson (University of California, San Diego) discusses the molecular evolution of leukemia stem cells during the session entitled "Cancer and Blood Disease."

cells in the brain: the cells have an amplification of the EGF receptor gene and the centromere of chromosome 7, two typical mutations of different glioblastomas variants. ${ }^{17}$ Tabar and colleagues isolated $\mathrm{CD} 133^{+}$cells from human glioblastoma samples and cultured them in vitro to test their ability to generate tumor vessels. Interestingly, they found that after seven days in vitro, $\mathrm{CD} 133^{+}$cancer stem cells differentiate into blood vessel cells expressing CD105, CD31, and CD34, which are typical endothelial markers in the brain. Tabar's group confirmed this in vivo by inoculating NOD/SCID mice with $\mathrm{CD} 133^{+} \mathrm{GFP}^{+}$cells isolated from glioblastoma tumors. Their results showed that gliobastomas that developed in NOD/SCID mice were characterized by $\mathrm{GFP}^{+}$blood vessels cells, suggesting that the $\mathrm{CD}_{133^{+}}$cancer stem cells were able to differentiate in vivo into endothelial cells necessary for tumor self-maintenance. Together, these data provide new insights for the development of novel drugs or therapies for treating glioblastomas that aim at inhibiting the endothelial transition of $\mathrm{CD} 133^{+}$cancer stem cells.

The third speaker of the session was Catriona Jamieson (University of California, San Diego; Fig. 2). Her talk explored the molecular evolution of leukemia stem cells (LSCs) during progression of chronic myeloid leukemia (CML) from the chronic phase $(\mathrm{CP})$ to blastic phase (BP). Several studies have shown that CML is characterized by the Philadelphia $(\mathrm{Ph})$ chromosome, which has a BCR/ABL1 rearrangement with enhanced kinase activity. ${ }^{18}$ Although it has been assumed that the BCR/ABL1 mutation occurs first in hematopoietic stem cells (HSCs) that are responsible for the development of the CML-CP phase, ${ }^{19}$ little is known about the molecular mechanisms that trigger the disease to transition to the more severe CML-BP phase. Using RNA sequencing and nanoproteomics approaches, Jamieson identified splice variants and point mutations that commonly occur in all CML tumor samples processed. These candidate genes are grouped into three classes: (1) genes involved in LSC aberrant self-renewal (such as Jak2), (2) genes involved in LSC dormancy (Shh), and (3) genes that promote LSC survival ( $\mathrm{Bcl} 2)$. Using inhibitors against these genes, Jamieson presented preliminary in vitro results: (1) TG101348, a Jak2 inhibitor, when combined with desatinib, a BCR/ABL1 inhibitor, arrested stem cell self-renewal ability; (2) Shh inhibitors were able to reactivate the stem cell cycle transition from G0 to G1; and (3) sabutoclax, a pan-inhibitor of the Bcl2 family, induced apoptosis of LSCs, thus arresting the blast phase. Taken together, these preliminary results support the possible use of these target genes for development of novel therapeutics aimed at inhibiting CML-BP development. 


\section{Keynote address}

The first invited keynote speaker was Irving L. Weissman (Stanford University; Fig. 3), whose work on hematopoiesis has been essential for defining the stages of development from hematopoietic stem cells (HSC) to their mature progeny. Many years of work on hematopoietic tissue, much of it led by Weissman's laboratory, has characterized phenotypically and genotypically nearly every step of differentiation of stem cells to mature progeny. Some of this basic science knowledge has been essential in understanding how normal hematopoietic cells become leukemic cells. For example, Weissman and colleagues have shown that despite preleukemic progression (such as EML1/ETO translocation) in HSCs, accumulation of further events in the progenitor compartment is responsible for the formation of leukemic stem cells (LSC). ${ }^{20}$ Weissman's group identified the cell marker CD47, which is upregulated in mouse and human acute myeloid leukemia (AML) stem cells and functions as a "don't eat me signal." Weissman's group demonstrated that expression of CD47 on a human leukemia cell line improves tumor engraftment in immunodeficient mice due to evasion by CD47-expressing tumor cells of phagocytosis. ${ }^{21}$ This suggests that cancer stem cells can defeat programmed cell removal by upregulating CD47 on their surface, which has led the way to a new anticancer treatment: blocking CD47 with an antibody so that phagocytosis can eliminate tumor cells, for example, AML stem cells. ${ }^{22}$ Selective targeting of tumor cells by CD47 antibody is explained by the simultaneous presence on tumor cells, but not on most normal cells, of calreticulin, which acts as a prophagocytic signal. ${ }^{23}$ Furthermore, the combination of CD47 antibody with rituximab, a monoclonal antibody that engages $\mathrm{Fc}_{\mathrm{C}}$ receptors on NK cells and macrophages, has a synergistic action that results in total elimination of human non-Hodgkin lymphomas in mice. ${ }^{24}$ Further studies on bladder, ovarian, and breast cancers suggest that different tumors use the same mechanism to escape immunosurveillance and can therefore be attacked by anti-CD47 treatment. This is a great example of translational research based on the evolution from basic science to the development of new drugs against human cancers.

\section{Repairing our heart and muscles}

The skeletal and cardiac muscle tissues constitute a large portion of the human body and are characterized by a set of different conditions that affect their

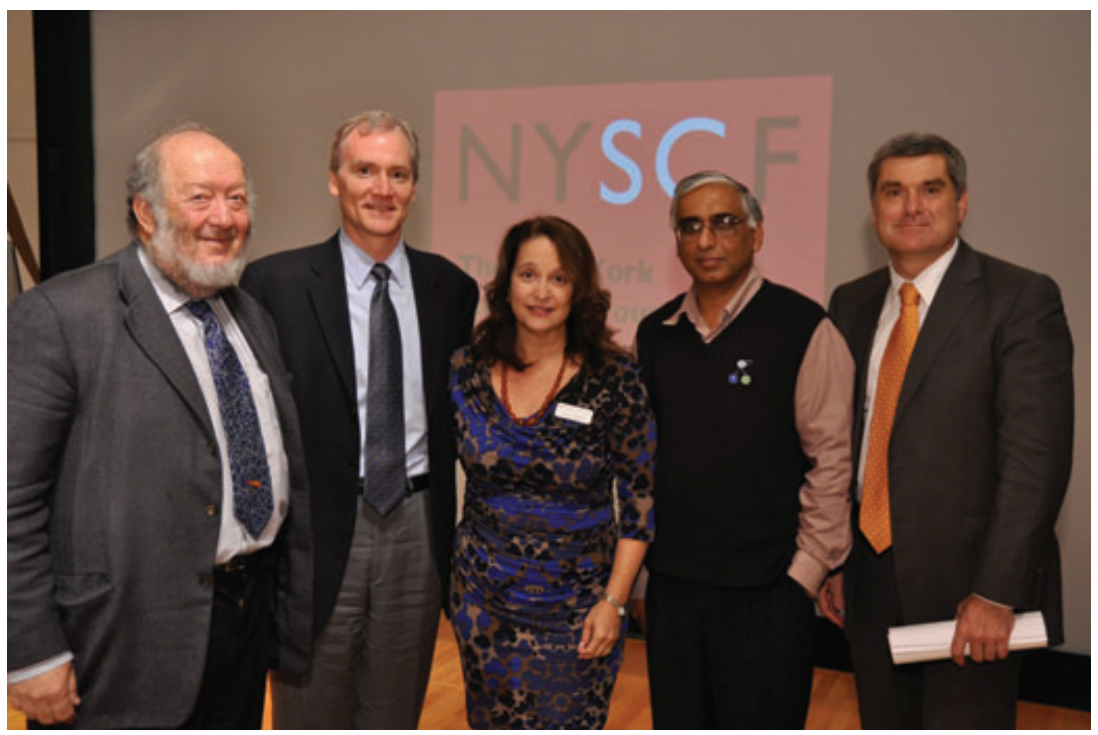

Figure 3. Irving L. Weissman (Stanford University and NYSCF Medical Advisory Board Member), Marc Tessier-Lavigne (The Rockefeller University and NYSCF Medical Advisory Board Member), Susan L. Solomon (CEO, New York Stem Cell Foundation), Mahendra S. Rao (Center for Regenerative Medicine, National Institutes of Health, and NYSCF Medical Advisory Board Member), and Craig B. Thompson (Memorial Sloan Kettering Cancer Center and NYSCF Medical Advisory Board Member) gather after a panel discussion entitled "The Future of Regenerative Medicine." 
functionality. The understanding of the molecular mechanisms governing the development, regeneration, and repair of these tissues is fundamental for developing innovative therapeutic solutions for the treatment of a large variety of medical conditions associated with disability and death.

During her presentation Margaret Buckingham (Institut Pasteur) addressed the role of satellite stem cells in skeletal muscle regeneration, as well as the function of Pax genes and downstream targets in different stages of tissue development and regeneration. Satellite cells, quiescent cells found between the basement membrane and the sarcolemma of individual muscle fibers, undergo profound transcriptional changes upon activation. Microarray analysis of in vivo quiescent and activated cells reveals how satellite cells protect from oxidative damage, maintain quiescence, and are primed for activation when proper signals are provided. ${ }^{25}$ This finding sheds light on the importance of extracellular matrix degradation for satellite cell migration and activation and demonstrates that upregulation of proteinases is crucial for optimal tissue regeneration in vivo. Buckingham's group has focused on the expression of Pax 3 and its regulation of the myogenic determination factor Myf5 during development and regeneration. Genetic analysis and chromatin immunoprecipitation studies reveal that Pax3 specifically binds a conserved sequence upstream of Dmrt2 and regulates its expression. During tissue development, Dmrt2 regulates the early activation of the Myf5 gene (Myf5), which plays a central role in the formation of the first skeletal muscle in the somite. ${ }^{26}$ In contrast, Myf5 is already transcribed in satellite stem cells of adult muscle before the onset of myogenesis, although translation into a functional protein is repressed via binding of microRNA-31 and subcellular sequestration of the microRNA-RNA complex in ribonucleoprotein granules. Consistent with this expression of microRNA-31, antagonists of microRNA-31 in animal models of injury promoted tissue regeneration and increased fiber size. Upon activation, this post-transcriptional repression is released and cells undergo myogenic differentiation. ${ }^{27}$ Buckingham highlighted the importance of silencing and sequestering tissue-specific regulatory gene transcripts to ensure rapid responses to tissue damage, and how similar control mechanisms may be involved in the regeneration of other somatic tissues.
Next, Deepak Srivastava (University of California, San Francisco) discussed his group's efforts to develop novel therapeutic strategies for human cardiac disorders based on known developmental pathways. By studying key molecular events during heart development, Srivastava's lab has identified a cascade of transcription factors and microRNAs that regulate early differentiation of cardiac progenitors and, later, their expansion into ventricular chambers. ${ }^{28-36}$ Many of these pathways can be used to guide differentiation of pluripotent stem cells into cardiac, endothelial, and smooth muscle cells that may be useful for regenerative medicine. One example of translating these observations into regenerative approaches is the group's recent success in direct reprogramming of cultured mouse postnatal cardiac fibroblasts ( $\mathrm{CFs}$ ) into cardiomyocytelike cells using a combination of three core developmental transcription factors, Gata4-Mef2C-Tbx5 (GMT). ${ }^{37}$ These induced cardiomyocytes (iCMs) express cardiac-specific markers, have a global gene expression profile similar to neonatal cardiomyocytes, and exhibit spontaneous $\mathrm{Ca}^{2+}$ flux, electrical activity, and contraction. Compared to iPSC reprogramming, reprogramming of CFs to iCMs with GMT occurs more rapidly (starting at day 3) and with a higher efficiency, up to $20 \%$. Furthermore, Srivastava's unpublished data confirm that this technique also works in vivo. Using a Cre-mediated lineage tracing technique, his group has demonstrated that resident CFs can be reprogrammed into iCMs through retrovirus-mediated GMT transduction of mouse heart. Interestingly, the in vivo reprogramming efficiency was greater than that observed in vitro; and iCMs exhibited properties, such as connexin 43 (Cx43) localization, ultrastructure under an electron microscope, action potential, and contractility, that closely resemble the native adult cardiomyocytes, suggesting a more complete reprogramming than in vitro. Most importantly, reprogrammed iCMs found in the scar zone formed after myocardium infarction resulted in improved cardiac function. Given that CFs normally compose over $50 \%$ of all the cells in the heart, these findings raise the possibility of reprogramming the vast pool of endogenous CFs into functional cardiomyocytes for regenerative purposes.

Michael Rudnicki (Ottawa Health Research Institute; Fig. 4) presented work on the identification of signaling pathways that regulate the function of 


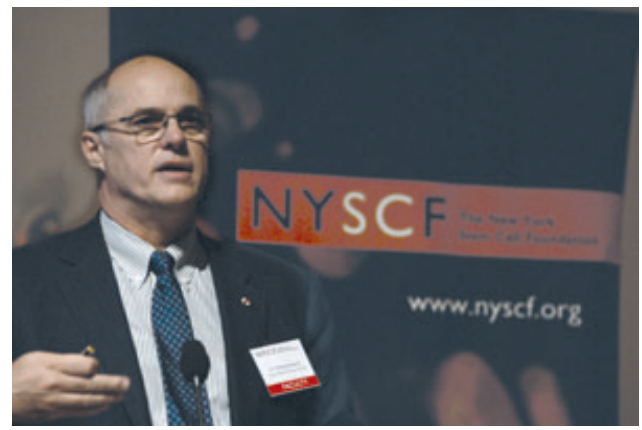

Figure 4. Michael Rudnicki (Ottawa Health Research Institute, Canada) presents his latest work with molecular regulation of muscle stem cell function.

satellite stem cells in adult skeletal muscle. Satellite stem cells support growth, homeostasis, and regeneration of skeletal muscle tissue through asymmetric (apical-basal) and symmetric (planar) divisions. ${ }^{38}$ His laboratory has found that satellite cells uniformly express the transcription factor Pax7. ${ }^{39}$ However, studies in mice have demonstrated that $10 \%$ of cells within the Pax7 population are negative for Myf5. Pax $7^{+} \mathrm{Myf5}^{-}$cells were found to give rise to $\mathrm{Pax}^{+} \mathrm{Myf5}^{+}$cells through asymmetric division within the satellite cell niche, indicating a positional control of cell fate. In agreement with this, transplantation studies revealed that the $\mathrm{Pax} 7^{+} \mathrm{Myf5}^{+}$cells are a subpopulation of myogenic progenitors that preferentially differentiate, whereas $\mathrm{Pax} 7^{+} \mathrm{Myf5}^{-}$cells contribute to satellite niche repopulation. ${ }^{40}$ The ability of $\mathrm{Pax} 7^{+} \mathrm{Myf5}^{-}$ cells to expand the satellite pool suggests that these cells may have therapeutic potential in treating a large variety of degenerative disorders affecting the skeletal muscle system. Furthermore, Rudnicki described the mechanism through which Pax7 activity is regulated upon asymmetric cell division, resulting in Myf5 transcription. Tandem affinity purification and mass spectroscopy analysis led to the identification of a set of cofactors interacting with Pax7, including the Wdr5-Ash2LMLL2 histone methyltransferase complex, which directs chromatin modification and allows the transcription of myogenic determination genes. ${ }^{41}$ Similar studies have led to identification of additional Pax7 interacting proteins, such as protein arginine $N$-methyltransferase-4 (CARM1/PRMT4). CARM1 binds Pax7 and regulates its function, through methylation of N-terminal arginines, which is necessary for the recruitment of the Wdr5Ash2L-MLL2 complex. Mutation of the methylation sites in the Pax7 sequence reduces the ability of Pax7 to upregulate Myf5 transcription, indicating a direct control of myogenic specification by CARM1 in the satellite compartment. Rudnicki also presented new findings demonstrating the common embryonic origin of brown fat and skeletal muscle. Isolated $\mathrm{Pax}^{+} \mathrm{Myf5}^{+}$satellite cells were found to differentiate toward the adipogenic lineage when appropriately stimulated. The transcription factor PDRM16 was found to control the bidirectional fate switch between skeletal myoblasts and brown fat cells. Knockdown of PDRM16 in brown fat precursor results in increased expression of myogenic genes, whereas ectopic expression of PDRM16 in myoblasts induces brown fat differentiation. Furthermore, immunopurification studies followed by mass spectroscopy demonstrate that PDRM16 interacts with PPAR- $\gamma,{ }^{42}$ recognized as a master transcriptional regulator of adipogenesis. ${ }^{43}$ These data indicate a direct control of PDRM16 on brown fat differentiation.

\section{Neurodegeneration and spinal cord injury}

Steven Goldman (University of Rochester Medical Center), the first speaker of the session entitled "Neurodegeneration and Spinal Cord Injury," presented recent work on a new strategy to isolate human oligodendocyte progenitor cells (OPCs) from fetal human brain. ${ }^{44}$ Following previously published data from his lab, in which OPCs were isolated by FACS based on the expression of the cell surface ganglioside marker A2B5, Goldman's group identified that OPCs can be further enriched by isolating cells expressing the receptor CD140a (PDGFRa), a subpopulation of the $\mathrm{A} 2 \mathrm{~B}^{+}{ }^{+}$cells. ${ }^{45}$ $\mathrm{CD}_{140 \mathrm{a}^{+}}$cells from human fetal brain, depleted of neuronal- and astrocytic-specific markers, are able to migrate and myelinate neuronal axons when transplanted into the hypomyelinated mouse brain. Myelination was faster and more efficient than that observed after transplantation of $\mathrm{A} 2 \mathrm{~B} 5^{+}$cells that were not further enriched for CD140a expression. This finding holds true when human $\mathrm{CD} 140 \mathrm{a}^{+}$cells are transplanted in chemically induced demyelinating rat brains and when OPCs are produced from human iPS cells, though with variable efficiency rates. Finally, Goldman presented interesting unpublished data from experiments with the 
shiverer mouse model following transplantation of $\mathrm{CD}_{140 \mathrm{a}^{+}}$human OPCs into the mouse brains. The cells gave rise to myelinating oligodendrocytes and astrocytes, and the brains were found to be chimeric, with $>70 \%$ of the glial cells being of human origin (8 months posttransplantation), suggesting that human donor cells outpace host-derived cells. Furthermore, the transplanted cells retained a human phenotype and function with high synaptic potentiation. Interestingly, Pavlovian-based behavioral tests show that the chimeric mice are better at learning by association and can be conditioned to fear faster than their wild-type counterparts, a sign that these mice might be "smarter." In addition to allowing the study of basic biological interactions of human glial cells, this strategy provides a unique mouse model in which to study diseases of the central nervous system, including human-specific infectious diseases.

In the second talk of the session, Paul Tesar (Case Western Reserve University) presented recently published work on the differentiation of mouse epiblast stem cells (EpiSCs) to OPCs. ${ }^{46}$ Tesar and his group are attempting to produce pure populations of OPCs by directed differentiation, using the signals that normally play important roles during normal development of oligodendrocytes, and to identify the required developmental transitions to produce functional OPCs. In the first stage, pluripotent EpiSCs are specified to the neuroectodermal lineage and cells are organized into typical neural rosette structures by blocking the activin-nodal and BMP signaling pathways for four days. In the second stage, the neuroectodermal-like cells are patterned to regionspecific neural precursor cells by addition of retinoic acid and $\mathrm{SHH}$ for one day. In the final stage, production of OPCs results from platelet-derived growth factor (PDGF) and FGF signaling after an additional five days. This ten-day differentiation protocol results in a population of highly enriched cells expressing OPC-specific markers that display the typical bipolar morphology of in vivo counterparts. Such mouse EpiSC-derived OPCs can be expanded for at least eight passages-results consistent across four independent EpiSC lines. After a further four days, the OPCs differentiate to highly specific mature oligodendrocytes (lacking any neuronal or astrocytic markers) that express basic myelin protein. The functionality of these OPCs was tested by injecting them into forebrain slices from the hypomyelinated shiverer mouse. In this context, the cells produced oligodendrocytes that migrated and myelinated neuronal axons in the host mouse brain tissue. Tesar reported that similar experiments are under way to find analogous methodologies that will robustly produce OPCs from human iPS cells. Finally, Tesar presented data (submitted) involving direct conversion of fibroblasts to OPCs, with a 10 $15 \%$ efficiency; the OPCs could expand, differentiate to mature oligodendrocytes, and myelinate sections of shiverer mouse brain.

Clive Svendsen (Cedars-Sinai Regenerative Medicine Institute) presented two approaches to treating Huntingon's disease (HD) and other neurodegenerative disorders. The first part of his talk summarized his lab's work over the previous 14 years using fetal brain tissue transplant approaches as a therapeutic intervention for HD and Parkinson's disease (PD) models. ${ }^{47,48}$ This method includes isolation of human progenitors from the fetal cortex and then passage of the cells by a "chopping method" to insure cell-cell contact. After 20 passages, these progenitors can differentiate, at a high percentage, to astrocytes. Such astrocyte progentitors, injected into animal models, produce human astrocytes that take 120 days to mature. The therapeutic potential of these cells can be amplified using ex vivo gene therapy enabling the cells to produce the neuroprotectant glial cell-derived neurotrophic factor. This combination is effective in modulating the deleterious effects observed in the N171-82Q mouse model of HD1. Svendsen described attempts to move this approach to clinical application for a variety of neurologic disorders, including HD, ALS, macula degeneration, and stroke. The second part of Svenden's talk described the progress of the iPSC Stem Cell Consortium for Huntington's disease, which is a collaboration between investigators from multiple research institutions, including Svendsen's, to generate and characterize HD patient-specific iPSCs. This unpublished work, funded by an NIH Grand Opportunities Grant, provides an opportunity for analysis of the HD iPSCs by groups with different expertise, as well as independent validation of experimental results by different laboratories. iPSCs have been generated from patients with different levels of CAG repeats in the Huntington gene. The number of CAG repeats dictates whether a person will develop HD and positively correlates with age of onset. ${ }^{49}$ Various labs within the consortium have demonstrated numerous disease-related 
phenotypes for neurons generated from multiple clones of higher CAG repeat iPSCs, including delays in neuronal maturation, adhesion properties, ATP metabolism, and cell death in response to stresses such as acute pulses of glutamate and BDNF withdrawal. Sensitivity to glutamate is also associated with decreased ability to reset calcium homeostasis, which may explain why HD neurons are more sensitive to excitotoxic insults.

\section{NYSCF-Robertson Prize lecture}

During the NYSCF-Robertson Prize lecture, Peter J. Coffey (University College London; Fig. 5) presented an overview of the work he has done with the London Project to Cure Blindness to find a treatment for age-related macular degeneration (AMD). AMD affects roughly 14 million older adults in the United States and in Europe. Currently, dry AMD, which affects $90 \%$ of the clinical population, has no known therapeutic treatment. Wet AMD, which affects $10 \%$ of the clinical population, can be treated with anti-VEGF therapy, but this therapy is expensive and time consuming, as patients must receive monthly injections of drugs into the eye.

In wet AMD, blood vessels, which perfuse blood into the retina, protrude through a weakened Bruch's membrane, the membrane dividing the choroid and the retinal pigment epithelial (RPE) cell layer. ${ }^{50}$ The protrusion of these blood vessels into the RPE cell layer compromises its function and cuts off the nutrition and structural support it provides for the photoreceptors, eventually leading to detachment of the macula and vision loss. ${ }^{51}$ In order to treat wet AMD, Coffey and colleagues have developed a macular translocation surgery in which the macula is reconstructed via its rotation. Macular translocation resulted in $25 \%$ of patients gaining three lines of acuity up to three years after surgery. ${ }^{52}$ However, this surgery is very complex and time consuming, and the cost per patient is exceedingly high. Therefore, Coffey and colleagues attempted macular translocation with $360^{\circ}$ retinotomy and autologous RPE-choroid patch graft, which has dramatically reduced costs as well as potential surgical complications, such as trauma to the photoreceptors themselves. However, RPE-choroid patch grafts were found to be inferior to macular translocation alone, partially because exogenous RPE grafts lacked sufficient metabolic support and the graft membrane did not allow sufficient attachment of the grated cells to the Bruch's membrane..$^{53}$ To address the problem of exogenous transplantation rejection, Coffey's group was first able to develop RPE cells generated from hESCs and successfully transplant these cells into the RCS rat model with inherited retinal degeneration. His group showed that photoreceptors could

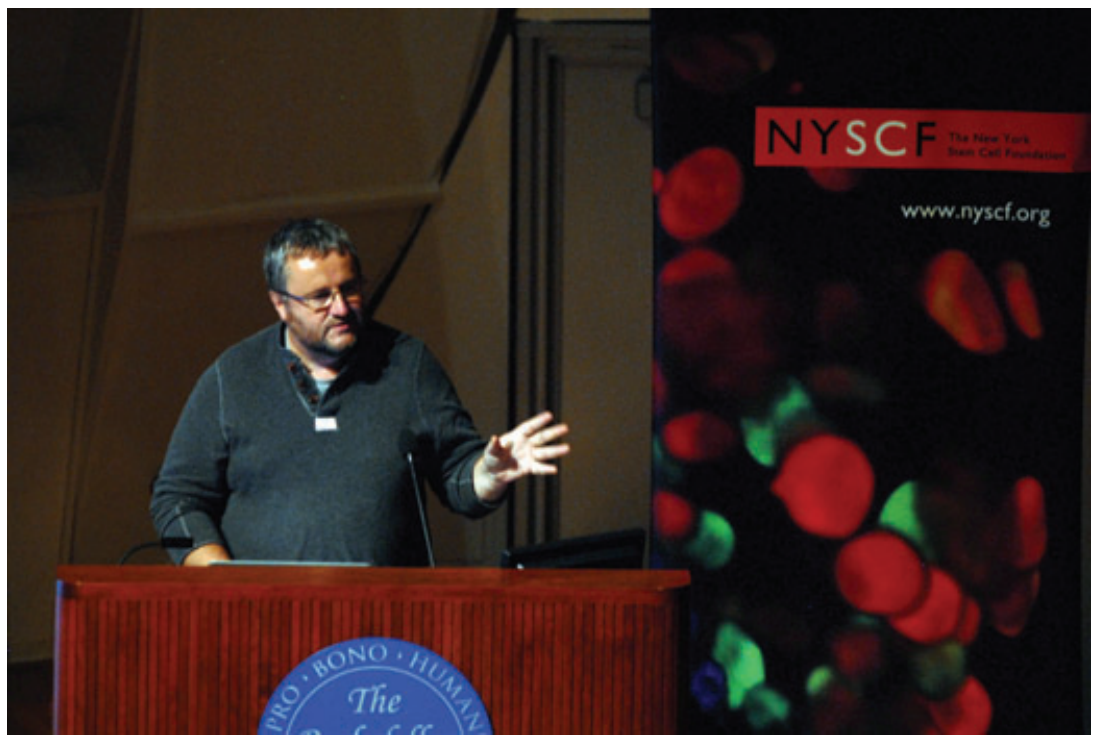

Figure 5. Peter J. Coffey (University College London, the London Project to Cure Blindness, and recipient of the Inaugural NYSCF-Robertson Prize in Stem Cell Research) delivers a special keynote address on his use of human ESCs to cure age-related macular degeneration. 
be rescued using both electrophysiological and behavioral tests, such as pattern discrimination and head tracking after implantation of hESC-derived RPE cells. ${ }^{54,55}$ Indeed, grafts of RPE cells derived from iPS cells into RCS rats induce the short-term maintenance of photoreceptors and significantly increase visual acuity. ${ }^{56}$ Coffey reported that experiments using choroid patch grafts from RPE-hESCs in pig retina result in the proliferation of functional photoreceptors with normal autofluorescence, indicating that the insertion of choroid patch grafts from RPE-hESCs into human eyes shows significant promise in the treatment of wet AMD. Phase I/II clinical trials based on this research are proposed for 2012 .

\section{Programming and reprogramming}

Dieter Egli (New York Stem Cell Foundation; Fig. 6), the first speaker of the session entitled "Programming and Reprogramming," reported on two recent publications aimed at generating patient-specific stem cells through the use of somatic cell nuclear transfer (SCNT). Egli first highlighted growing concerns about iPS cells, stressing the need for continued work in SCNT. ${ }^{57-59}$ However, many labs have failed to fulfill the potential of SCNT, thus posing the question of whether these failures are because of the preliminary nature of the studies, or because of an intrinsic issue with the human egg that prevents reprogramming.
Beginning with his experience at Harvard University, Egli highlighted the issues of recruiting oocyte donors when only "altruistic donors" can be enrolled. ${ }^{60}$ After moving to the New York Stem Cell Foundation, Egli's study continued in collaboration with Columbia University Medical Center's Center for Women's Reproductive Care (CWRC), where, to date, 16 donors have participated. Initial experiments corroborated previous findings but also suggested that developmental failure was due to transcriptional dysfunction. Using microarray analysis, the expression profile of such failures closely resembled oocytes treated with transcriptional inhibitor aminitin, raising the following questions: (1) Is artificial activation of the oocyte causing this arrest? This was not the case, as parthenogenetic blastocysts could be made. (2) Is oocyte manipulation causing this arrest? This was answered no by demonstrating that if the genome was removed and replaced into the same oocyte, parthenogenetic blastocysts could still form. (3) Is the somatic cell genome interfering with early development, or is it the removal of the oocyte genome? To answer this, a somatic cell (GFP labeled) was fused to an oocyte with an intact oocyte genome. Following activation, development to the blastocyst stage demonstrated GFP activation and, hence, that the somatic cell genome had participated in blastocyst development. Stem cells derived from the blastocysts developed into all three germ layers and expressed markers typical of stem cells.

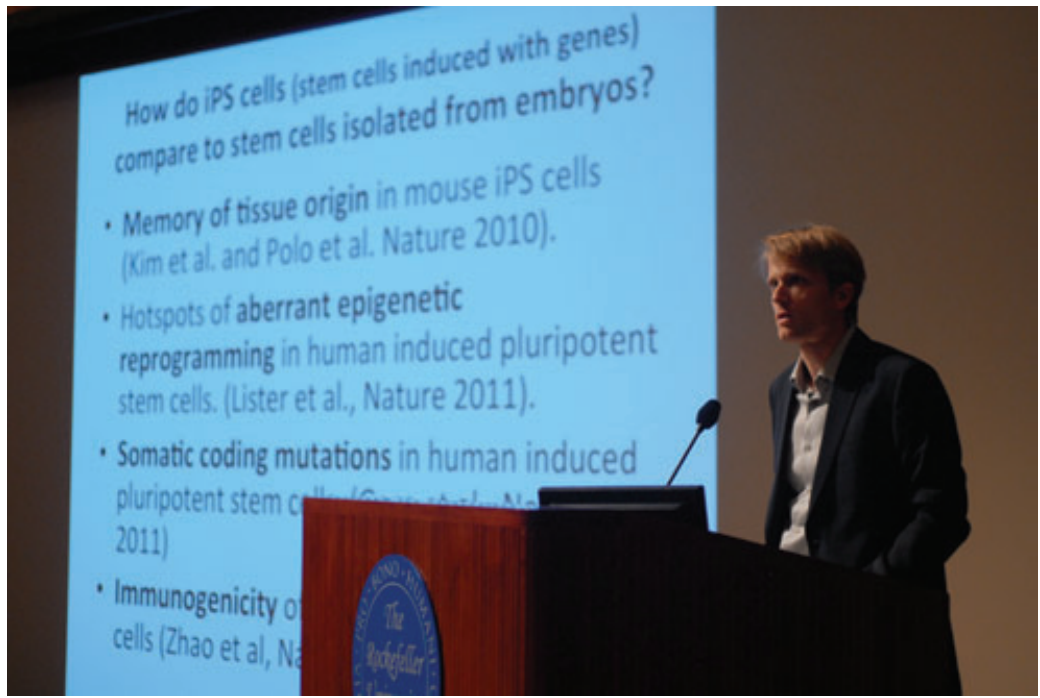

Figure 6. Dieter Egli (the New York Stem Cell Foundation) shares his groundbreaking work on reprogramming after nuclear transfer in the session entitled "Programming and Reprogramming." 
However, these stem cells were karyotypically abnormal, with a triploid genome. To eliminate the issue of timing, the oocyte genome was removed at the first interphase, which led to developmental arrest. Sequencing analysis of both gDNA and cDNA was undertaken with an allelic ratio of $\sim 0.6$ found in both, suggesting that the somatic genome was as active as the oocyte genome. Analysis of gene expression profiles of the stem cells showed that there was no preferential expression of either fibroblast or stem cell genes, suggesting that no "somatic memory" was present. ${ }^{61}$ This work suggests that the oocyte can reprogram the somatic genome, and further work is now under way to identify what causes developmental arrest following removal of the oocyte genome.

Alex Meissner (Harvard University; Fig. 7) began his talk by referencing the same issues that Egli raised concerning iPS cells. While acknowledging that critical investigation is warranted, Meissner pointed out that there is also a wide range in variation among ES cells lines. These variations were shown by methylation mapping, gene expression profiling, and use of a quantitative differentiation assay. Furthermore, while some iPS cell lines do not cluster with ES lines in these analyses, many do, suggesting that they are as useful as many ES lines. ${ }^{62}$ Meissner went on to discuss DNA methylation dynamics and how to further our understanding of where and when DNA methylation is used as a regulatory mechanism. He described unpublished data that highlights the critical role of DNA methyla- tion during development. Zygotes from fertilized mouse oocytes were collected at multiple times during development. It was initially found that although there were few differences in global methylation patterns between E7.5 and adult tissue, analysis of the oocyte itself revealed a globally lower level of methylation than that in sperm. Following fertilization, a continuous shift in demethylation (of the paternal genome) is seen until E6.5 at implantation. Furthermore, the inner cell mass (ICM) of the blastocyst appears to differ greatly from that of the somatic genome in which classically low CpG dense areas are highly methylated. It was also found that for a number of genes, including Dnmt1, separate maternal and somatic methylation patterns exist. Until the eight-cell stage of development, the somatic Dnmt1 CpG island is highly methylated before switching to a demethylated state, with the opposite being true for the maternal version. From unpublished, ongoing, collaborative work with Egli, the initial analysis of mouse nuclear transfer zygotes appears to show that nuclear transfer blastocysts show comparable global demethylation to that seen in zygotes. It appears, however, that similar to sperm, the oocyte is capable of removing some methylation from previously highly methylated areas, although a number of regions remain as methylated as in the somatic cell. Therefore, remodeling by the oocyte does take place; however, not all regions are remodeled equally.

Kevin Eggan (New York Stem Cell Foundation and Harvard University) ended the programming

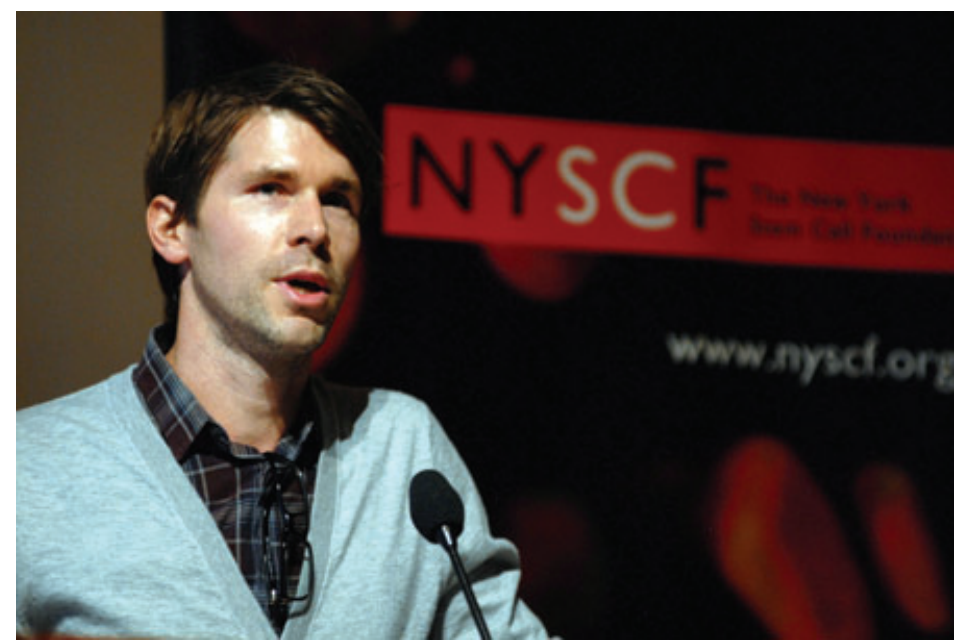

Figure 7. Alex Meissner (Harvard University) discusses his progress in epigenetic reprogramming. 
and reprogramming session by discussing his recently published work describing the direct conversion of mouse and human fibroblasts into induced motor neurons (iMNs) by a process referred to as transdifferentiation. ${ }^{63}$ The purpose of this study was to generate amyotrophic lateral sclerosis (ALS) disease-specific motor neurons by transdifferentiation, which subsequently could be compared to similarly derived control motor neurons. Eggan, who pioneered the first ALS-specific iPS cells that could make disease-specific motor neurons, ${ }^{64}$ suggested that iMNs, owing to their significantly faster generation time, could provide a quicker preview of disease-specific phenotypes than iPS approaches. Eggan's group first attempted to generate iMNs by virally overexpressing eight transcription factors alone, or in combination, known to be important for either the motor neuron differentiation process or in the mature motor neuron itself. This approach was unsuccessful in generating iMNs from fibroblasts obtained from HB9:GFP mice (which produce GFP-fluorescing motor neurons). However, when Eggan's group combined this strategy with the three transcription factors used by Marius Wernig's group to create more general neurons from fibroblasts (iNs), ${ }^{65}$ they successfully generated iMNs. This could be reduced to a six or seven transcription factor cocktail, and could also be used to create iMNs from human fibroblasts. Similar to iNs, ${ }^{66}$ the transdifferentiation process to iMNs does not appear to involve a neural progenitor intermediate. iMNs were shown to resemble bona fide motor neurons by a variety of criteria, including gene expression studies, electrophysiological properties, formation of cholingeric synapses with muscle cells generated from the $\mathrm{C} 2 \mathrm{C} 12$ cell line, and implantation into the developing chick spinal cord with subsequent appropriate axonal migration to peripheral targets. Eggan finished his talk by showing preliminary unpublished data suggesting that human iMNs prepared from ALS patients with genetic forms of the disease demonstrate increased electrical excitability compared to control iMNs, which could predispose the former to increased sensitivity to excitotoxic insults.

\section{Conclusion}

NYSCF's sixth annual meeting convened leaders in stem cell research, both senior and innovative young scientists, providing attendees with novel scientific presentations that focused on a wide variety of diseases and techniques. The speakers, including two scientists from NYSCF's inaugural class of NYSCFRobertson investigators, gave conference participants a first-hand look at unpublished and recently published pioneering research. The "Seventh Annual Translational Stem Cell Research Conference" will take place on October 10-11, 2012.

\section{Acknowledgments}

\section{New York Stem Cell Foundation}

Sixth Annual Translational Stem Cell Research Conference

\section{Co-chairs}

Lee Goldman, MD, MPH, Columbia University Medical Center

Antonio M. Gotto Jr., MD, DPhil, Weill Cornell Medical College

Douglas A. Melton, PhD, Harvard University

Allen M. Spiegel, MD, Albert Einstein College of Medicine

Marc Tessier-Lavigne, $\mathrm{PhD}$, The Rockefeller University

Craig B. Thompson, MD, Memorial Sloan-Kettering Cancer Center

\section{Scientific Co-chairs}

Moses V. Chao, PhD, Skirball Institute of Biomolecular Medicine

Zach W. Hall, PhD, The New York Stem Cell Foundation

Ihor Lemischka, PhD, The Mount Sinai School of Medicine

Dan R. Littman, MD, PhD, Skirball Institute of Biomolecular Medicine

\section{Principal Sponsor}

Robertson Foundation

\section{Co-sponsoring Institutions}

Albert Einstein College of Medicine

Columbia University Medical Center

Helen and Martin Kimmel Center for Stem Cell

Biology, New York University School of Medicine Mount Sinai School of Medicine

Tri-Institutional Stem Cell Initiative:

- Memorial Sloan-Kettering Cancer Center

- The Rockefeller University

- Weill Cornell Medical College

\section{Conflicts of interest}

The authors declare no conflicts of interest. 


\section{References}

1. Hall, Z.W. et al. 2010. Breaking ground on translational stem cell research. Ann. N.Y. Acad. Sci. 1189 (Suppl. 1): E1-E11.

2. Marshall, C. et al. 2011. The New York Stem Cell Foundation: Fifth Annual Translational Stem Cell Research Conference. Ann. N.Y. Acad. Sci. 1226: 1-13.

3. Green, M.D. \& H.W. Snoeck 2011. Novel approaches for immune reconstitution and adaptive immune modeling with human pluripotent stem cells. BMC Med. 9: 51.

4. Green, M.D. et al. 2011. Generation of anterior foregut endoderm from human embryonic and induced pluripotent stem cells. Nat. Biotechnol. 29: 267-272.

5. D'Amour, K.A. et al. 2006. Production of pancreatic hormone-expressing endocrine cells from human embryonic stem cells. Nat. Biotechnol. 24: 1392-1401.

6. Chen, S. et al. 2009. A small molecule that directs differentiation of human ESCs into the pancreatic lineage. Nat. Chem. Biol. 5: 258-265.

7. Thorel, F. et al. 2010. Conversion of adult pancreatic alphacells to beta-cells after extreme beta-cell loss. Nature 464: 1149-1154.

8. Thorel, F. et al. 2011. Normal glucagon signaling and $\beta$-cell function after near-total $\alpha$-cell ablation in adult mice. Diabetes. Epub.

9. Ding, B.S. et al. 2011. Endothelial-derived angiocrine signals induce and sustain regenerative lung alveolarization. Cell 147: 539-553.

10. Butler, J.M. et al. 2010. Endothelial cells are essential for the self-renewal and repopulation of Notch-dependent hematopoietic stem cells. Cell Stem Cell 6: 251-264.

11. Hooper, A.T. et al. 2009. Engraftment and reconstitution of hematopoiesis is dependent on VEGFR2-mediated regeneration of sinusoidal endothelial cells. Cell Stem Cell 4: 263-274.

12. Kobayashi, H. et al. 2010. Angiocrine factors from Aktactivated endothelial cells balance self-renewal and differentiation of haematopoietic stem cells. Nature Cell Biol. 12: 1046-1056.

13. Ding, B.S. et al. 2010. Inductive angiocrine signals from sinusoidal endothelium are required for liver regeneration. Nature 468: 310-315.

14. Butler, J.M. et al. 2010. Instructive role of the vascular niche in promoting tumour growth and tissue repair by angiocrine factors. Nat. Rev. Cancer 10: 138-146.

15. Singh, S.K. et al. 2004. Identification of human brain tumor initiating cells. Nature 432: 396-401.

16. Wang, R. et al. 2010. Glioblastoma stem-like cells give rise to tumor endothelium. Nature 468; 829-833.

17. Verhaak, R.G. et al. 2010. Integrated genomic analysis identifies clinically relevant subtypes of glioblastoma characterized by abnormalities in PDGFRA, IDH1, EGFR and NF1. Cancer Cell 17: 98-110.

18. Cross, N.C. et al. 2008. BCR-ABL1-positive CML and BCRABL1-negative chronic myeloproliferative disorders: some commons and contrasting features. Leukemia 22: 19751989.

19. Bruns, I. et al. 2009. The hematopoietic stem cell in chronic phase CML is characterized by a transcriptional profile re- sembling normal myeloid progenitor cells and reflecting loss of quiescience. Leukemia 23: 892-899.

20. Miyamoto, T. et al. 2000. AML1/ETO-expressing nonleukemic stem cells in acute myelogenous leukemia with 8;21 chromosomal translocation. Proc. Natl. Acad. Sci. USA 97(13): 7521-7526.

21. Jaiswal, S. et al. 2009. CD47 is upregulated on circulating hematopoietic stem cells and leukemia cells to avoid phagocytosis. Cell 138(2): 271-285.

22. Majeti, R. et al. 2009. CD47 is an adverse prognostic factor and therapeutic antibody target on human acute myeloid leukemia stem cells. Cell 138(2): 286-299.

23. Chao, M.P. et al. 2010. Calreticulin is the dominant prophagocytic signal on multiple human cancers and is counterbalanced by CD47. Sci Transl Med 2(63): 63ra94.

24. Chao, M.P. et al. 2010. Anti-CD47 antibody synergizes with rituximab to promote phagocytosis and eradicate nonHodgkin lymphoma. Cell 142(5): 699-713.

25. Pallafacchina, G. et al. 2010. An adult tissue-specific stem cell in its niche: a gene profiling analysis of in vivo quiescent and activated muscle satellite cells. Stem Cell Res 4(2): 77-91.

26. Sato, T. et al. 2010. A Pax3/Dmrt2/Myf5 regulatory cascade functions at the onset of myogenesis. PLoS Genet 6(4): e1000897.

27. Buchan, J.R. et al. 2009. Eukaryotic stress granules: the ins and outs of translation. Mol Cell 36(6): 932-941.

28. Garg, V. et al. 2003. GATA4 mutations cause human congenital heart defects and reveal an interaction with TBX5. Nature 424: 443-447.

29. Bock-Marquette, I. et al. 2004. Thymosin beta4 activates integrin-linked kinase and promotes cardiac cell migration, survival and cardiac repair. Nature 432: 466-472.

30. Garg, V. et al. 2005. Mutations in NOTCH1 cause aortic valve disease. Nature 437: 270-274.

31. Zhao, Y. et al. 2005. Serum response factor regulates a muscle-specific microRNA that targets Hand2 during cardiogenesis. Nature 436: 214-220.

32. Zhao, Y. et al. 2007. Dysregulation of cardiogenesis, cardiac conduction, and cell cycle in mice lacking miRNA-1-2. Cell 129: 303-317.

33. Fish, J.E. et al. 2008. miR-126 regulates angiogenic signaling and vascular integrity. Dev. Cell 15: 272-284.

34. Ivey, K. N. et al. 2008. MicroRNA regulation of cell lineages in mouse and human embryonic stem cells. Cell Stem Cell 2: 219-229.

35. Cordes, K.R. et al. 2009. miR-145 and miR-143 regulate smooth muscle cell fate and plasticity. Nature 460: 705-710.

36. Ieda, M. et al. 2009. Cardiac fibroblasts regulate myocardial proliferation through betal integrin signaling. Dev. Cell 16: 233-244.

37. Ieda, M. et al. 2010. Direct reprogramming of fibroblasts into functional cardiomyocytes by defined factors. Cell 142: 375-386.

38. Kuang, S. et al. 2007. Asymmetric self-renewal and commitment of satellite stem cells in muscle. Cell 129(5): 999-1010.

39. Seale, P. et al. 2000. Pax7 is required for the specification of myogenic satellite cells. Cell 102(6): 777-786.

40. Darabi, R. et al. 2011. Assessment of the myogenic stem cell compartment following transplantation of 
Pax3/Pax7-induced embryonic stem cell-derived progenitors. Stem Cells 29(5): 777-790.

41. McKinnell, I.W. et al. Pax7 activates myogenic genes by recruitment of a histone methyltransferase complex. Nat. Cell Biol. 10(1): 77-84.

42. Rosen, E.D. et al. 1999. PPAR gamma is required for the differentiation of adipose tissue in vivo and in vitro. Mol. Cell 4(4): 611-617.

43. Seale, P. et al. 2008. PRDM16 controls a brown fat/skeletal muscle switch. Nature 454(7207): 961-967.

44. Sim, F.J. et al. 2011. CD140a identifies a population of highly myelinogenic, migration-competent and efficiently engrafting human oligodendrocyte progenitor cells. Nat. Biotechnol. 29(10): 934-941.

45. Windrem, M.S., et al. 2008. Neonatal chimerization with human glial progenitor cells can both remyelinate and rescue the otherwise lethally hypomyelinated shiverer mouse. Cell Stem Cell 2(6): 553-565.

46. Najm, F.J. et al. 2011. Rapid and robust generation of functional oligodendrocyte progenitor cells from epiblast stem cells. Nat. Methods 8: 957-962.

47. Ebert, A.D. et al. 2010. Ex vivo delivery of GDNF maintains motor function and prevents neuronal loss in a transgenic mouse model of Huntington's disease. Exp. Neurol. 224: 155162.

48. Capowski, E.E. et al. 2007. Lentiviral vector-mediated genetic modification of human neural progenitor cells for ex vivo gene therapy. J. Neurosci. Methods 163: 338349.

49. Roos, R.A.C. 2010. Huntington's disease: a clinical review. Orphanet. J. Rare Dis. 5: 40.

50. Simpson, E. 1986. Immune regulation. Immunol. Today 7: 1.

51. Lu, B. et al. 2009. Long-term safety and function of RPE from human embryonic stem cells in preclinical models of macular degeneration. Stem Cells 27: 2126-2135.

52. Chen, F.K. et al. 2010. Increased fundus autofluorescence associated with outer segment shortening in macular translocation model of neovascular age-related macular degeneration. Invest. Ophth. Vis. Sci. 51(8): 4207-4212.
53. Chen, F.K. et al. 2009. A comparison of macular translocation with patch graft in neovascular age-related macular degeneration. IOVS 50(4): 1848-1855.

54. Coffey, P.J. et al. 2002. Long-term preservation of cortically dependent visual function in RCS rats by transplantation. Nat. Neurosci. 5(1): 53-56.

55. Gias, C. et al. 2007. Preservation of visual cortical function following retinal pigment epithelium transplantation in the RCS rat using optical imaging techniques. Eur. J. Neurosci. 25(7): 1940-1948.

56. Carr, A.J. et al. 2009. Protective effects of human iPS-derived retinal pigment epithelium cell transplantation in the retinal dystrophic rat. PLOS ONE 4(12).

57. Gore, A. et al. 2011. Somatic coding mutations in human induced pluripotent stem cells. Nature 471(7336): 63-67.

58. Lister, R. et al. 2011. Hotspots of aberrant epigenomic reprogramming in human induced pluripotent stem cells. Nature 471(7336): 68-73.

59. Ohi, Y. et al. 2011. Incomplete DNA methylation underlies a transcriptional memory of somatic cells in human iPS cells. Nat. Cell Biol. 13(5): 541-549.

60. Egli et al. 2011. Impracticality of Egg Donor Recruitment in the Absence of Compensation. Cell Stem Cell 9(4): 293-294.

61. Noggle, S. et al. 2011. Human oocytes reprogram somatic cells to a pluripotent state. Nature 478(7367): 70-75.

62. Bock, C. et al. 2011. Reference maps of human ES and iPS cell variation enable high-throughput characterization of pluripotent cell lines. Cell 144(3): 439-452.

63. Son, E.Y. et al. 2011. Conversion of mouse and human fibroblasts into functional spinal motor neurons. Cell Stem Cell 9: 205-218.

64. Dimos, J.T. et al. 2008. Induced pluripotent stem cells generated from patients with ALS can be differentiated into motor neurons. Science 321: 1218-1221.

65. Vierbuchen, T. et al. 2010. Direct conversion of fibroblasts to functional neurons by defined factors. Nature 463: 10351041.

66. Qiang, L. et al. 2011. Directed conversion of Alzheimer's disease patient skin fibroblasts into functional neurons. Cell 146: 359-371. 